\title{
Mathematicians and Primary School Teachers Learning From Each Other
}

Jason Cooper and Abraham Arcavi (both Weizmann Institute of Science, Rehovot, Israel)

1 MATHEMATICIAN1: How do I prove the formula for the area of triangles that look like this? How can I convince myself that it's half the base times the altitude?

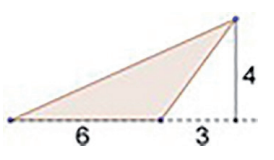

[Teachers work in small groups for approximately 5 minutes]

2 MATHEMATICIAN1: Does anyone object to a hint?

3 TEACHER: No hints!!!

4 MATHEMATICIAN1:Questions like this can fascinate kids. This is what's fun in math. Not calculating areas.

[5 minutes later]

5 TEACHER: If you don't solve it now, I'll work on it all night.

6 MATHEMATICIAN1: The big triangle's area is $6+3$ - its base - times 4 over 2. I know this from what we showed previously about right-angled triangles. Now, we take away the smaller triangle: 3 times 4 over 2 . We can do algebra. $S=\frac{(6+3) \times 4}{2}-\frac{3 \times 4}{2}=\frac{(6+3-3) \times 4}{2}$.

You can actually see the algebraic trick visually; the area of this is... [pause] Well, I guess you can't really see it. We show it by algebraic proof.

7 TEACHER: But I want to do it as something tangible.

8 MATHEMATICIAN1: I don't see how you can do that.
9 TEACHER: Look. If I take two congruent triangles, I can form a parallelogram. Its area is exactly the rectangle's - the base multi-

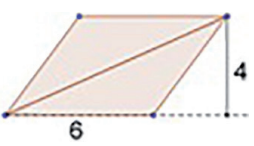
plied by its altitude.

10 MATHEMATICIAN1: Very nice! But you're assuming you know the formula for the area of a parallelogram

11 TEACHER: But I can move from the parallelogram to a rectangle. Cut here and move it to here, and I get a rectangle.

12 MATHEMATICIAN1: Will that work for any parallelogram?

13 TEACHER: Of course!

14 MATHEMATICIAN1: Are you sure? Even if the base is very small and the side is very long?

15 MATHEMATICIAN2: In this case, you can slice the parallelogram. If you slice it thinly enough, each parallelogram will be of the "right" kind. I just thought of it now. If I hadn't been here, I'd never have thought of it!

This transcript is taken from a professional development course for practising primary school teachers (grades 
3-6) in Israel, which was initiated and run by mathematicians. The idea of mathematicians being involved in the professional development of teachers should not appear far-fetched to the readers of this newsletter; Felix Klein believed that "the whole sector of mathematics teaching, from its very beginnings at elementary school right through to the most advanced level research, should be organized as an organic whole" (Klein 1923, p. 24). From this perspective, it is natural to assume that university mathematicians should have an important role in the professional development of primary school teachers, though in Israel, and in many other contexts, the involvement of mathematicians in primary school mathematics is rare. There are significant differences between the mathematics taught and practised in universities and in primary schools and, though research mathematicians generally have some experience of teaching mathematics, their university experience may be of limited relevance in the context of teaching in primary school. Hyman Bass, a former president of the American Mathematical Society, who has become extensively involved in teacher education, suggests that "Mathematics for Teaching" is best seen as a field of applied mathematics and that "the first task of the mathematician who wishes to contribute in this area is to understand sensitively the domain of application, the nature of its mathematical problems, and the forms of mathematical knowledge that are useful and usable in this domain" [Bass 2005]. However, it is far from obvious how mathematicians should go about engaging in Mathematics for Teaching and gaining such a "sensitive understanding" of its issues.

The aim of this article is to describe, by means of two representative examples, how this meeting of mathematicians and teachers can create opportunities not only for primary school teachers to learn mathematics but also for mathematicians to learn some Mathematics for Teaching. We will highlight some differences between the two communities' perspectives on teaching and learning mathematics and show how these differences provide a springboard for mathematical and pedagogical discussions, which create opportunities for mutual learning - from and with each other. For a more comprehensive account of professional development, the reader is referred to the first author's unpublished doctoral dissertation (Cooper, 2016) and to published work (Cooper \& Karsenty, 2016; Pinto \& Cooper, 2017; Cooper \& Arcavi, 2013).

\section{Background on the course}

In 2009, Raz Kupfermann, a professor of mathematics at the Hebrew University of Jerusalem, approached the Ministry of Education with an initiative to undertake a professional development course (henceforth PD) for practising primary school teachers. He had taken an interest in mathematics teaching at his children's school and sensed that teachers might benefit from the perspective of mathematicians in order to deepen their understanding of the content they teach. Thus, he suggested a for-credit course that would focus on a deep understanding of primary school content. This was an unusual stance since, in Israel (as in many other countries), pri- mary school PD is usually taught by experienced teachers and is pedagogically oriented, attending to issues such as textbook selection, "best practices" for teaching particular topics, methods for managing heterogeneity in classrooms, where to find high quality supplemental tasks, etc. The assumption that underlies this stance is that primary school content is straightforward and does not warrant special attention. The ministry officials were happy to offer PD that focuses on the mathematics of primary school and consented to have it run by mathematicians. Teacher feedback was so positive that a group of graduate students were recruited to address the high enrolment in the following years.

In 2010, Kupfermann approached the two authors (separately), sensing that the PD might benefit from the involvement of mathematics educators. This involvement evolved into the first author's doctoral project, under the guidance of the second author. Data was collected in the 2011-2012 school year, in which 100 teachers participated in six separate groups, each of which was co-taught by two graduate students at the Hebrew University (for the most part, PhD students of mathematics) under the aegis of Kupfermann. The first author was a participant observer, witnessing and contributing to the planning of PD lessons and debriefing the instructors after the lessons. We took field notes and audio recordings from all the lessons (ten 3-hour lessons for each of the six groups), audio recordings of meetings and interviews with the instructors, teacher expectation questionnaires (at the outset) and feedback questionnaires (after each lesson). When possible, teachers were also interviewed. The doctoral dissertation was a multiple case study of episodes from 10 of the 60 recorded lessons. Here, we focus on a short section from one such episode, which was not included in the dissertation.

\section{Analysis of the excerpt - What was going on?}

In this section, we take a close look at some of the utterances from the excerpt and hypothesise as to the parties' underlying perspectives (implicit or even tacit) on the nature of mathematical activity and on its teaching and learning in primary school.

A recurring theme in the PD was the instructors' attempts to present the learning of mathematics as a sense-making activity, knowing that the Ministry of Education's curriculum, and its implementation in textbooks, may lead teachers to over-emphasise procedural aspects of the subject. The Ministry of Education requires that 5 th grade students should "calculate the area of polygons, including obtuse-angle triangles" [The Pedagogical Secretariat of the Israeli Ministry of Education, 2009A]. Most textbooks explain the formula for a triangle's area, first for right-angled triangles (half a rectangle) and then for interior altitudes (dropping an altitude to separate the triangle into two right-angled triangles). Though students are expected to apply the formula for all triangles, students, and for the most part teachers as well, do not usually question why the formula should hold when the altitude is external. Raising this issue in the PD carries some implicit ideas about mathematics and its teaching 
and learning, which we propose as MP1 (MathematicianPrinciple-1), based on U1 and U4: ${ }^{1}$

MP1. Mathematics is about making sense of formulas, not just about applying them. Teachers should know why the formulas that they teach "work". Perhaps students should as well.

This sentiment is shared by Lockhart, who used the same problem of making sense of the formula for the area of a triangle in his well-known "Mathematician's Lament" (2009).

It is evident that the teachers were highly engaged in this investigation, at first refusing to receive any hints ("No hints!!!" - U3) yet eventually demanding resolution ("If you don't solve it now, I'll work on it all night." - U5). However, the teachers were not quite satisfied with the mathematician's explanation ("I want to do it as something tangible." - U7), implying that:

\section{TP1. Explanations for students should be tangible.}

It is difficult to say exactly what would count as a tangible explanation for teachers. In this context, based on familiar explanations for the formula when the altitude is internal, and also on U9 in the excerpt, it seems to be related to scissor-congruence, namely, a tangible explanation is one that can be demonstrated by cutting the triangle along straight lines and rearranging its parts (possibly duplicated). This notion of tangibility is grounded in the teacher's experience - knowing what kinds of activities primary school students engage in and what kinds of mathematical reasoning they find to be more (or less) appropriate. For a research mathematician, there may be little difference between adding and subtracting areas; however, for primary school students and teachers, it is much more natural to see a triangle as being made up of two right-angled triangles than it is to see a triangle's area as the difference between two areas. For the latter, one must envision a third triangle that is not part of the problem.

Another unexpected difficulty had to do with what Mathematician1 called an "algebraic trick" (in fact, an application of the distributive property) to obtain the formula for the triangle's area. Algebraic manipulation is not part of the primary school curriculum; students may be familiar with the distributive property but if so then only through appropriate visual mediation. For example, 2-digit multiplication, which relies strongly on the distributive property, is often mediated as the area of a rectangle, where the distributive property is represented by sectioning a rectangle (e.g. $3 \times 27$ is represented by two joined rectangles, one representing $3 \times 20$ and the other representing $3 \times 7$ ). However, Mathematician1 had to concede that in the case of the triangle's area, his "algebraic trick" (i.e. the distributive property) did not have an obvious visual representation (U6, U8).

$\mathrm{U} 1, \mathrm{U} 2$, etc. indicate the numbered utterances from the lesson excerpt.
A teacher suggested a very nice alternate explanation (forming a parallelogram from two congruent triangles), yet it raised some concerns on the part of the mathematician. Do we already "know" the area of a parallelogram (U10)? Does the argument address the general case or are we making some tacit assumptions (U12)? Though not voiced explicitly, perhaps he was concerned with circular logic, wondering whether the formula for the area of a parallelogram might later be proven based on the area of a triangle - apparently a more basic mathematical object. These concerns imply the following.

MP2. Since mathematical knowledge is built on previous knowledge, it is important to be clear about what is known and what is not known at any particular time, taking care to avoid circular logic.

MP3. Mathematical arguments should be general, i.e. valid for all cases.

Regarding MP2, we note that the mathematicians in the PD tended to use the words "what is known" differently from the teachers. For the mathematicians, the meaning was usually epistemic, a mathematical kind of knowing, referring to what has already been shown to be true. Teachers, on the other hand, often took a cognitive/pedagogical approach to "knowing", considering what their students had encountered in the past, regardless of how rigorously it had been justified. For example, teachers realise that students "know" halves well before the topic of fractions is introduced in school.

Both the mathematicians in this transcript drew on their mathematical experience. Mathematician1, concerned with MP3 (arguments should be general), spontaneously generated an example (U14) for which the teacher's argument would fail. This is the same example that the Gestalt theorist Max Werthheimer (1959) posed in a class where the teacher had taught a method for calculating the area of a parallelogram that follows the reasoning presented in U11. In that classroom, the teacher called the skewed parallelogram "a queer figure", which her students naturally could not deal with. Yet, for Mathematician1, a method that does not address such "queer" figures is inadequate. Mathematician2 suggested an argument to overcome this deficiency, reducing the ill-behaved parallelogram to a disjoint union of wellbehaved parallelograms.

\section{Rapprochement - what was learned}

The mathematicians and the teachers had different agendas regarding the PD. The mathematicians were concerned primarily with mathematical content (the area of a triangle) and meta-content (mathematics as sense-making), whereas the teachers had students and teaching in mind, perhaps looking out for an activity to use in their own classroom. In this section, we discuss the opportunities for learning that this episode afforded, for both the teachers and the mathematicians in creating a space where the agendas of both communities combined around a mathematical investigation, allowing productive communication and reflection on each other's points of view. 
Perhaps the most noteworthy aspect of this episode is the engagement and enthusiasm on the part of the teachers. Many of them had not previously had opportunities to experience the challenge and excitement of mathematical investigation and discovery and were thus unlikely to create such opportunities for their students. The concern they voiced regarding the tangible nature of explanations suggests that at least some of them were considering the possibility of taking this activity to their own classes, thus coming closer in their teaching to principle MP1, whereby doing mathematics is primarily a sensemaking activity. This sense-making has some rules: the teachers needed to apply principle MP2 and be explicit about how they "know" the area of a parallelogram. Furthermore, they needed to take care that this "knowing" applies to all parallelograms. This short episode can be seen as a demonstration of what the university perspective on mathematics is about and how it can be applied to reveal the relevance of this kind of mathematics for primary school.

The mathematicians were provided with an opportunity to reflect on the tangible and visual nature of geometric proofs invoked by a primary school teacher. Mathematician1 seems to have been quite surprised to discover that his application of the distributive rule was not represented visually in his sketch (U6) and was a bit too quick in resigning to the necessity of an abstract proof ("I don't see how you can do [something tangible]"). It was a teacher, drawing on her own mathematical knowledge for teaching, who suggested a proof that is more appropriate for primary school, avoiding the necessity to imagine the difference between two areas. The mathematicians accepted this constraint and drew on their own expertise (perhaps taking inspiration from notions of "slicing" that they have encountered in the context of Cavalieri's principle or in the context of integration) to complete a tangible yet general proof. Mathematician2's comment is revealing - his "discovery" of a proof was a result of his interaction with the teachers - he would not have come to realise it on his own.

\section{The plot thickens: volume of a pyramid}

In one of the following PD sessions, the same mathematicians decided to address a related topic - the volume of a pyramid. We describe this lesson more briefly, contrasting it with the case of the triangle.

According to the Ministry of Education's guidelines for sixth grade, the volume of a pyramid - one third the volume of a prism with the same base and altitude "will be deduced by filling hollow prisms and pyramids with water or sand" [The Pedagogical Secretariat of the Israeli Ministry of Education, 2009B]. Teachers are instructed to demonstrate ${ }^{2}$ that it takes three pyramids to fill a prism with the same base and altitude as the pyramid. We may wonder whether such a demonstration satisfies principle MP1. Though they agreed that such a demonstration is better than simply providing the for-

\footnotetext{
2 Perhaps a more appropriate word than "deduce" from the Ministry's guidelines.
}

mula, the mathematicians did not see it as a mathematical justification.

How can primary school teachers and students "make sense" of the formula and understand why it "works"? Can the formula be explained in a manner that will be "tangible"? The volume of a pyramid (one third the volume of a prism with the same base and altitude) appears to be a natural extension of the area of a triangle (one half the area of a rectangle with the same base and altitude) but, for some reason, $1 / 2$ is replaced by $1 / 3$. And, indeed, some teachers were expecting a tangible explanation that follows the case of the triangle, perhaps breaking the pyramid into disjoint parts that can be duplicated and rearranged as a prism, and asked why the 3-dimensional case should be qualitatively different from the planar case.

The mathematicians knew that there is, in fact, a deep difference. They were familiar with Hilbert's third question from 1900: "Given any two polyhedra of equal volume, is it always possible to cut the first into finitely many polyhedral pieces that can be reassembled to yield the second?", and also with Dehn's negative answer to this question, showing that there exist tetrahedra with equal base area and equal altitude (hence equal volume) that have different Dehn invariants and thus are not scissorcongruent. Given this state of affairs, together with the teachers' expectation for a tangible explanation (TP1), a new notion of tangibility was called for.

The mathematicians opted for a demonstration but not the one suggested by the Ministry. They decided to focus on a special case: three identical "right-angle" pyramids that can be arranged to form a cube. They led the teachers through an activity that involved solving the tangible puzzle of constructing a cube from pyramids. This, too, is only a demonstration, not an explanation of "why" the formula holds in general and certainly not

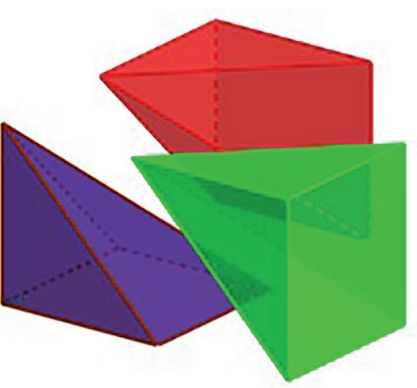

Cube comprised of 3 pyramids. a proof of the general case. Yet, it has some special features - both mathematical and pedagogical - that made it more appealing for the mathematicians than the Ministry's suggestion.

- Pedagogical: It suggests a classroom-ready activity for students - teachers can use it, perhaps with some modifications, to actively engage students in their own classrooms, as opposed to the demonstration advocated by the Ministry of Education, where students passively observe the teacher. The activity could even include constructing the pyramids from printouts of their nets. - Mathematical: Having teachers calculate the dimensions of the net of the pyramid, ostensibly in order to prepare handouts for a classroom activity, provided an opportunity for them to engage with some relatively advanced mathematics (from the perspective of primary school teachers) that they don't often deal with: 
the Pythagorean theorem, in an authentic problem-solving setting.

- Mathematical: It is not difficult to prove that a cube can be dissected into three disjoint pyramids, assuming familiarity with the cube's rotational symmetries; rotations around the cube's diagonal Net of a pyramid. map the three pyramids onto each other.

- Mathematical ${ }^{3}$ : This special case can, in principle, be extended to the general case, relying on the following observations.

- A general pyramid can be approximated by a collection of disjoint pyramids, each having a square base and all having a common vertex. Thus, it is sufficient to prove the volume formula for pyramids with square bases.

- Applying Cavalieri's principle, it can be shown that all pyramids with congruent bases and equal altitudes have the same volume, since their planar sections parallel to the base have the same area. Thus, without loss of generality, it can be assumed that the edge from the pyramid's vertex to its base is perpendicular to the base.

- Given the base, a pyramid's volume is proportional to its altitude. This, too, is a consequence of Cavalieri's principle: if a pyramid's altitude is multiplied by a factor of $\mathrm{k}$, keeping the base unchanged, and the pyramid is then dilated by a factor of $1 / \mathrm{k}$ (multiplying its volume by a factor of $\left.(1 / \mathrm{k})^{3}\right)$, the result is, by Cavalieri's principle, a pyramid whose volume is $(1 / \mathrm{k})^{2}$ times the volume of the original, since each section has $(1 / \mathrm{k})^{2}$ times the area of the respective section in the original. The conclusion is that the first transformation (multiplying the altitude by a factor of k) must have multiplied the volume by a factor of $\mathrm{k}$.

Although some of these observations may be a bit difficult to comprehend, they have simple and familiar versions for triangles in the plane, which can help make sense of the 3-dimmensional case.

In this demonstration, the mathematicians had found a way to reconcile their principle of making sense of formulas (MP1) with the teachers' principle of tangibility (TP1), using their knowledge of advanced mathematics (Dehn invariant) to propose a demonstration that is not only pedagogically appropriate but also mathematically valid, without seriously compromising their principle of generality (MP3).

\section{In conclusion}

A number of research mathematicians have taken an active interest in pre-college mathematics education and, in particular, in teacher preparation and professional development. Some have shared their experience with

3 Based, in part, on personal communication with Hyman Bass. other mathematicians (e.g. the March 2011 issue of the Notices of the American Mathematical Society), reporting that elementary mathematics is surprisingly interesting and engaging. In this article, we have tried to add to this body of work and to exemplify the mutual benefits of a meeting of mathematicians and primary school teachers in professional development. In such a meeting, the teachers clearly have much to learn from the mathematicians. What is less obvious is that the mathematicians may also have much to learn, not only about teaching mathematics in primary school but also about the nature of the mathematics that is taught and learnt. In this meeting, both sides were committed to their own agenda; the mathematicians were guided by universal principles of mathematics that should not be "watered down", even in primary school, and the teachers were guided by their expectation that the professional development should be relevant for their teaching, taking into account the children's ways of thinking and doing. Both parties remained true to their agendas, while at the same time listening sensitively to the other. As a result, the teachers had the opportunity to engage in mathematical investigation and discovery in the context of the content they teach, while the mathematicians had the opportunity to develop Mathematics for Teaching, along with a sensitivity for the nuances of mathematics as it is taught and learnt in primary school. On the way, they co-developed some new insights into teaching some mathematics that is often overlooked in primary school.

We have limited our discussion to two related episodes, yet they are representative of the whole of the PD. The first author's doctoral dissertation [Cooper 2016], in which 10 cases were analysed in depth, addressed no more than $10 \%$ of the data collected in a single year of this ongoing project, which was incredibly rich in opportunities for learning similar to the ones described above. This setting can serve not only as an opportunity for teachers' professional development but also as a way to address Bass' call for mathematicians, who wish to contribute in this area of school mathematics, to come to "understand sensitively the domain of application, the nature of mathematical problems, and the forms of mathematical knowledge that are useful and usable" [Bass 2005] in the context of primary school mathematics education. We have shown not only how such sensitivity can be developed but also how it can contribute to making mathematicians' expertise relevant for primary school teachers.

\section{References}

Bass, H. (2005). Mathematics, mathematicians, and mathematics education. Bulletin (New Series) of the American Mathematical Society, 42(4), 417-430.

Cooper, J. (2016). Mathematicians and primary school teachers learning from each other. Unpublished doctoral dissertation. Retrieved from https://stwww1.weizmann.ac.il/wp-content/uploads/2016/09/ Cooper-dissertation-finalAppendix.pdf.

Cooper, J., \& Karsenty, R. (2016). Can teachers and mathematicians communicate productively? The case of division with remainder. The Journal of Mathematics Teacher Education (First Online 17 October 2016). doi:10.1007/s10857-016-9358-7. 
Cooper, J., \& Arcavi, A. 2013. Mathematicians and elementary school mathematics teachers - meetings and bridges. In Y. Li, \& J. N. Moschkovich (Eds.), Proficiency and beliefs in learning and teaching mathematics - Learning from Alan Schoenfeld and Günter Törner (pp. 179-200). Rotterdam: Sense Publishers.

Klein, F. (1923). Göttinger Professoren. Lebensbilder von eigener Hand. 4. Mitteilungen des Universitätsbundes Göttingen, 5(1),11-36.

Lockhart, P. (2009). A Mathematician's Lament: How School Cheats Us Out of Our Most Fascinating and Imaginative Art Form. Bellevue Literary Press.

Pinto, A., \& Cooper, J. (2017). In the pursuit of relevance - Mathematicians designing tasks for elementary school teachers. International Journal of Research in Undergraduate Mathematics Education, 3(2), 311-337. doi:10.1007/s40753-016-0040-3.

The Pedagogical Secretariat of the Israeli Ministry of Education. (2009 A). Details of the curriculum in mathematics for grade 5. Retrieved from Mathematics for primary school for all sectors: http://meyda. education.gov.il/files/mazkirut_pedagogit/matematika/tochnyotlemud/kita5.pdf.

The Pedagogical Secretariat of the Israeli Ministry of Education. (2009 B). Details of the curriculum in mathematics for grade 6 . Retrieved from Mathematics for primary school for all sectors: http://meyda. education.gov.il/files/mazkirut_pedagogit/matematika/tochnyotlemud/kita6.pdf.

Wertheimer, M. (1959). Productive Thinking (enlarged edition). New York: Harper (first edition published 1945).

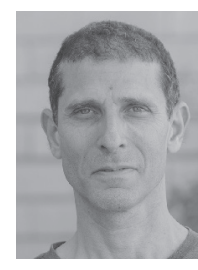

Jason Cooper is a research fellow at the University of Haifa's Faculty of Education. He is also a researcher at the Weizmann Institute's Department of Science Teaching. His research concerns various aspects of teacher knowledge, including roles of advanced mathematical knowledge in teaching and contributions of research mathematicians to the professional development of teachers. He has been a member of the ERME board since 2015.

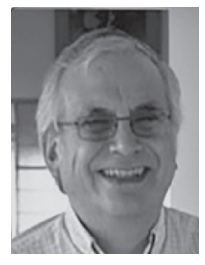

Abraham Arcavi is the incumbent of the Lester B. Pearson Professorial Chair at the Department of Science Teaching of the Weizmann Institute of Science, Israel. He works on the teaching and learning of mathematics, curriculum development and professional development of mathematics teachers. At present (2016-2020) he is the Secretary General of the International Commission on Mathematical Instruction (ICMI).

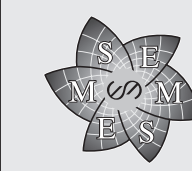

\section{New journal published by the} European Mathematical Society
European Mathematical Society Publishing House Seminar for Applied Mathematics

ETH-Zentrum SEW A21, CH-8092 Zürich, Switzerland subscriptions@ems-ph.org / www.ems-ph.org

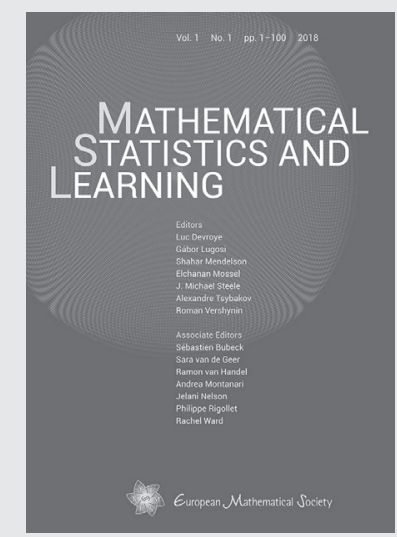

ISSN print $\quad 2520-2316$

ISSN online $2520-2324$

2018. Vol. 1. 4 issues

Approx. 400 pages.

$17.0 \times 24.0 \mathrm{~cm}$

Price of subscription:

$198 €$ online only /

$238 €$ print+online

\section{Editors:}

Luc Devroye (McGill University, Montreal, Canada)

Gabor Lugosi (UPF Barcelona, Spain)

Shahar Mendelson (Technion, Haifa, Israel and Australian National University,

Canberra, Australia)

Elchanan Mossel (MIT, Cambridge, USA)

J. Michael Steele (University of Pennsylvania, Philadelphia, USA)

Alexandre Tsybakov (CREST, Malakoff, France)

Roman Vershynin (University of Michigan, Ann Arbor, USA)

\section{Associate Editors:}

Sebastien Bubeck (Microsoft Research, Redmond, USA)

Sara van de Geer (ETH Zurich, Switzerland)

Ramon van Handel (Princeton University, USA)

Andrea Montanari (Stanford University, USA)

Jelani Nelson (Harvard University, Cambridge, USA)

Philippe Rigollet (MIT, Cambridge, USA)

Rachel Ward (University of Texas, Austin, USA)

\section{Aims and Scope}

Mathematical Statistics and Learning will be devoted to the publication of original and high-quality peer-reviewed research articles on mathematical aspects of statistics, including fields such as machine learning, theoretical computer science and signal processing or other areas involving significant statistical questions requiring cuttingedge mathematics. 